\title{
Étude des tours pronominaux et de l'épisode du groseillier d'après le corpus CLEA
}

Comment appréhender les corrélations langue-discours dans des productions verbales enfantines?

Study of the pronominal turns and the gooseberry episode from the corpus CLEA

- How to understand the language-speech correlations in children's verbal productions?

\section{Caroline Masseron}

\section{(2) OpenEdition}

\section{Journals}

Édition électronique

URL : http://journals.openedition.org/pratiques/4049

DOI : $10.4000 /$ pratiques.4049

ISSN : 2425-2042

Éditeur

Centre de recherche sur les médiations (CREM)

Référence électronique

Caroline Masseron, «Étude des tours pronominaux et de l'épisode du groseillier d'après le corpus

CLEA », Pratiques [En ligne], 177-178 | 2018, mis en ligne le 20 juillet 2018, consulté le 02 mai 2019.

URL : http://journals.openedition.org/pratiques/4049; DOI : 10.4000/pratiques.4049

Ce document a été généré automatiquement le 2 mai 2019.

(c) Tous droits réservés 


\section{Étude des tours pronominaux et de l'épisode du groseillier d'après le corpus CLEA}

Comment appréhender les corrélations langue-discours dans des productions verbales enfantines?

Study of the pronominal turns and the gooseberry episode from the corpus CLEA

- How to understand the language-speech correlations in children's verbal productions?

Caroline Masseron

1 L'étude procède en deux étapes, distinctes mais - nous l'espérons - complémentaires, qui s'appuient sur la dichotomie langue-langage, telle que la conçoivent notamment F. François, C. Hudelot et É. Sabeau-Jouannet (1984).

2 Au cours d'une première étape, en langue, nous explorons la question du système - le codage - des verbes pronominaux dans la langue et leur réalisation dans le corpus. En effet, suffisamment variés et fréquents d'une séance à l'autre, de complexité variable et soumis à des facteurs diversifiés (lexicaux, sémantiques, morphologiques et syntaxiques), les tours pronominaux (s'appeler, s'endormir, s'accrocher, s'en aller, se mettre à courir, etc.) nous ont paru pouvoir constituer un analyseur intéressant pour appréhender le corpus du point de vue de l'acquisition de la langue et des degrés de maitrise atteints par les locuteurs.

3 La seconde étape, "langagière ", qu'on dirait aussi volontiers " discursive ", s'attache à décrire les conduites ou stratégies verbales et les échanges tels qu'ils sont transcrits tout au long des 12 séances. Plus précisément, nous appréhendons ce second volet du travail par la comparaison du traitement langagier d'un épisode particulier, celui du groseillier. La perspective est alors plus onomasiologique ou référentielle, dominée par deux procès concurrents, la fuite du lapin (s'enfuir) et l'obstacle provisoire à sa réussite, soit son ralentissement sous l'effet du bouton du gilet qui se prend dans le filet ${ }^{1}$. 
4 À l'issue de ces deux temps d'analyse, nous essayerons de discuter les conditions d'une complémentarité entre faits de langue et faits de discours, dans le but d'établir en quoi ce double point de vue peut renforcer notre connaissance des apprentissages langagiers.

\section{Cadre général, démarche d'analyse et hypothèses didactiques}

\section{Le texte source et les séances : bref rappel sur les conditions de recueil des données}

5 L'histoire de Pierre le lapin est lue par le maitre, sans que les élèves disposent à aucun moment de l'album illustré. L'album aurait permis la saisie instantanée de la situation d'après une image qu'il se serait agi de "traduire » en mots. Les séances de langage sont donc dominées par la reformulation de ce qui a été d'abord verbalisé, entendu, compris comme tel par les élèves et de ce qu'ils ont retenu. Ce que l'on ne peut pas appréhender c'est l'état de la mémoire (compréhension) de l'histoire, pour un élève particulier, au début, au cours de et à l'issue de la séance de langage.

Nous observons que le rappel de l'histoire par les élèves est dominé par une alternance du passé composé ( $\mathrm{S} 9-4^{2}$ : Monsieur Grégory il a pris un panier pour attraper Pierre et Pierre il a sauté dans l'arrosoir) et du présent (S9-8: la maman elle dit je dois m'absenter), auxquels s'ajoutent l'imparfait (S9-4: monsieur Grégory le cherchait partout) et le plus-que-parfait (S9-40: la maman elle avait dit que Pierre il a perdu tous ses habits ; S9-50: la maman elle avait dit d'aller dans le pré). Le texte source, lui, est au présent. La translation du repère énonciatif (du présent au passé composé) s'opère plus ou moins bien, selon les séances (le présent domine la séance 2, contre le passé composé de la séance 3), selon les épisodes (les recommandations de la mère lapin à ses enfants, au discours direct, sont majoritairement restituées au présent, vs l'évocation du civet de lapin, au passé composé). Le choix du passé composé ou du présent n'est pas indifférent aux difficultés potentielles que soulèvent certains tours pronominaux (il s'accroche vs il s'est accroché, *s'a accroché, S6-15), même si ce facteur demeure secondaire, la réalisation erronée étant isolée ${ }^{3}$. Nous y reviendrons.

7 Les limites de notre traitement des données sont que celui-ci est strictement manuel. Nous l'avons fait sur la base de la lecture des séances et de l'observation des faits de langue dans leur support écrit. Autrement dit, notre traitement a consisté en un repérage des formes et des séquences dans les transcriptions écrites puis en des regroupements divers (tel verbe comme s'absenter, les temps et les modalités, etc.). Nous avons dans un premier temps sélectionné l'environnement étroit et syntaxique des verbes pronominaux (S9-26: monsieur Grégory s'est vite retourné) ; puis nous avons isolé des extraits plus larges qui restituent l'épisode du groseillier (S9-11: monsieur Grégory l'avait un filet et Pierre son bouton était fait attraper au filet et puis les oiseaux ils ont dit Pierre Pierre vite sauve-toi juste à temps). Les extraits dits du groseillier figurent en annexe. Les deux volets indiqués, les verbes pronominaux et la séquence du groseillier, ont été notre fil conducteur principal et ils constituent les parties suivantes de notre contribution. Auparavant, nous revenons sur le cadre d'analyse retenu pour étudier les faits de langue recensés, puis sur les implications didactiques inhérentes à la situation de dialogue du dispositif. Nous 
exposons enfin l'hypothèse qui nous fait utiliser les tours pronominaux comme un indice de performance langagière qui se révèle plus ou moins efficiente.

\section{Cadre de l'analyse morphosyntaxique : lexique-grammaire, approche pronominale et construction verbale (Blanche-Benveniste). Recensement lexical et classement syntaxique des formes pronominales}

Pour commencer, nous livrons notre résumé de l'histoire, en recourant sciemment aux verbes pronominaux. La succession des procès est donnée chronologiquement (1). Trois séquences en marquent les étapes : l'avertissement de la mère, l'aventure du lapin dans le jardin de Monsieur Grégory et le retour à la maison. La liste des verbes pronominaux à l'infinitif assortis de données circonstancielles (personnages, espace-temps) permet d'observer les spécifications lexicales et grammaticales des verbes, la fonction narrative (au sens proppien de la place occupée par le procès dans le déroulement de l'histoire), et les difficultés d'emploi que ces verbes présentent potentiellement à des usagers adultes et enfants. Figure ci-dessous en gras « le sommet de l'histoire » (son « nœud »).

(1) Unités pronominales selon un traitement lexical et la progression narrative

(La maman des lapins) doit S'ABSENTER (S'ADRESSER À ses enfants, leur recommandant de NE PAS SE RENDRE dans le potager)

(Désobéissant, Pierre le lapin) S'AVENTURER ${ }^{4}$ (dans le potager)

(Monsieur Grégory, le jardinier) S'APPROCHER (de Pierre le lapin)

(Pierre le lapin) S'ENFUIR

(Pierre le lapin) SE CACHER (dans l'arrosoir, rempli d'eau glacée)

(Pierre le lapin) S'ENRHUMER

(Alerté par un éternuement, Monsieur Grégory) SE RETOURNER (vers Pierre le lapin)

(Pierre le lapin) SE REMETTRE À COURIR

(Le bouton du gilet de Pierre le lapin) SE PRENDRE (dans le filet du groseillier)

(Pierre le lapin) SE DÉBARRASSER (du vêtement resté accroché)

(Pierre le lapin) réussit à S'ÉCHAPPER (S'ENFUIR, SE SAUVER)

(De retour à la maison, Pierre le lapin) NE PAS SE SENTIR BIEN

(Pierre le lapin) SE PROMETTRE de ne plus désobéir

(Réconforté, Pierre le lapin) S'ENDORMIR

(Les autres enfants lapins) SE PARTAGER LES MÛRES

(La maman des lapins) S'INTERROGER (SE DEMANDER) sur ce qui S'ÊTRE PASSÉ

La série qu'on vient de parcourir couvre en réduction bon nombre des propriétés des verbes pronominaux. Sont ainsi représentées à la fois la productivité lexicale de verbes polysémiques qui ne sont qu'accessoirement pronominaux (adresser, rendre, approcher, cacher, retourner, promettre...) et la série plus fermée des verbes essentiellement pronominaux (s'absenter, s'aventurer, s'enfuir, s'enrhumer, s'échapper ${ }^{5}$ ). Est également présente une occurrence d'auxiliaire aspectuel (se remettre à courir). Par ailleurs, on aura noté que la valence du sujet est dans tous les cas sauf un (italique gras, ci-dessus) tenue par des rôles sémantiques d'agent animé (Pierre, Monsieur Grégory, la maman, les autres enfants lapins).

- Le verbe noyau régit - commande - un nombre déterminé d'arguments, qui sont des rôles sémantiques (agent, patient, datif, manière) et des places syntaxiques (sujet, objet, locatif) qui sont obligatoires ou non et dont les formes sont nominales ou pronominales, directes ou «obliques» (dans le potager). Par exemple, demander quelque chose à quelqu'un, d'une certaine 
manière ou avec l'intention de, peut voir le datif (à quelqu'un) renvoyer à la même personne que le sujet et devenir se demander quelque chose d'une certaine manière (dernière occurrence cidessus [1]). Autre exemple, se retourner est intransitif, à quelles conditions (normes ?) peut-il sélectionner une suite du type se retourner vers $X$ ? Cet $X$ est-il obligatoirement un animé ? Peut-on (se) retourner vers la gare, ou bien (se) retourne-t-on en direction de la gare, sans parler du tour archaïsant s'en retourner?

- Le verbe réalisé dans un énoncé est doté d'informations qu'on qualifiera de modalités auxiliées : devoir s'absenter, se mettre à courir, etc. Ces modalités donnent un point de vue sur le procès («l'action » représentée par la base lexicale du verbe) : sur la manière dont il est envisagé (l'obligation de s'absenter pour un personnage) ou sur son déroulement et c'est alors la phase du procès qui est considérée (son début avec se mettre à).

- Les verbes pronominaux constituent une classe de verbes morphologiquement repérable mais complexe et hétérogène. Ils présentent l'intérêt méthodologique de guider simultanément l'observation du corpus et la consultation des listes de fréquence (Catach, 1984) appliquées aux personnes des formes fléchies (je dois m'absenter vs je me suis aventuré). Ils favorisent une réflexion sur les propriétés communes à tous les verbes et les propriétés spécifiques des verbes pronominaux.

10 Le cadre linguistique que nous avons privilégié est celui dit de l'approche pronominale (Blanche-Benveniste et al., 1984). L'alliance des faits syntaxiques et de l'analyse du français parlé justifie notre choix. Nous reviendrons sur le classement des constructions pronominales tel que le proposent $C$. Blanche-Benveniste et son équipe.

\section{Cadre de l'analyse didactique : conduite du dialogue, caractérisation des échanges et approximations langagières}

11 Des échanges verbaux ordinaires doivent à la fois garantir la pertinence thématique et informationnelle et satisfaire la dynamique du dialogue, tout en demeurant économiques. Par ailleurs, la complexité de "ce dont on parle ", la complexité de l'objet du discours, intervient elle aussi pour brouiller la forme de l'énoncé, la perturber. En d'autres termes, les échanges verbaux se construisent, toujours, quels qu'en soient les locuteurs, sur des approximations langagières qui peuvent contrevenir aux réglages standards et attendus de telle construction, lesquelles approximations - éventuellement - portent atteinte à la compréhension, en réception, de la visée du discours.

12 Mais les échanges verbaux de ce corpus sont particuliers: l'âge des locuteurs et le contexte scolaire font que ces échanges ne s'apparentent évidemment pas à ceux d'une conversation ordinaire. Si ces échanges n'échappent pas aux approximations, ils sont surdéterminés par les paramètres scolaires, d'ordre langagier et pédagogique (la pédagogie de l'écoute [Péroz, 2010]; les attentes et représentations des maitres), qui engagent forcément le mode de dire, au moins autant que le dit.

\section{Hypothèse : la maitrise lexicale et syntaxique des tours pronominaux constitue un indicateur pertinent pour rendre compte des seuils d'apprentissage langagier}

13 Les apprentissages langagiers ont un corrélat qui est l'enseignement de la langue, lequel de son côté implique des programmes et une progression dans le choix des faits de langue à 
traiter, la conception de leur matière notionnelle et celle de leurs supports d'entrainement et des modalités de travail pédagogique.

Les tours pronominaux $d u$ français offrent une matière linguistique très intéressante pour présenter différentes formes en usage, des plus ordinaires aux plus savantes, des routines pragmatiques et conversationnelles du français parlé au français académique écrit. Citons pour mémoire et à titre d'exemple la série suivante (2, ci-dessous), que nous posons comme potentiellement illustrative des formes types et des genres de discours apparentés. On y a notamment recensé des tours locutionnels fréquents à l'oral (si ça se trouve, qu'est-ce qui se passe, séries 1 et 2), les verbes pronominaux qui construisent un tour auxiliaire, modal ou semi-passif (s'entendre dire, se voir attribuer, se laisser faire, séries 3 et 4), jusqu'à des constructions pronominales simples mais lexicalement recherchées (se répandre, s'orienter) passibles de genres écrits académiques (y compris les genres de la presse, série 5) :

(2) Variété des tours pronominaux relativement à la variation du sujet (P3)

1) - Si ça se trouve il (n') est pas là

- Ça se voit qu'il est malade

2) - Comment ça s'est passé hier

- Qu'est-ce qui s'est passé

- Comment ça se passe depuis qu'il est parti

- Comment ça se fait que tu es là

3) - Il s'écoute parler

- Il se laisse vivre

- Elle s'est fait couper les cheveux

- Il s'est fait disputer

4) - Il se laisse faire

- Il s'est remis à courir

- Il s'est arrêté de fumer

- On s'est vu trop beaux

- Il s'est vu attribuer de nouvelles fonctions

- Il s'est entendu reprocher son absence

5) - L'épidémie se répand

- Le soleil se montrera plus généreux à partir de jeudi

- La discussion s'oriente sur le choix du meilleur candidat

- L'ouvrage s'ouvre sur un rappel des principales références

La série (2) n'a d'autre utilité que de témoigner de la productivité du tour pronominal et de la variété des registres, sous la forme d'un inventaire de formes empiriquement classées. Le corpus oral de productions d'enfants de 4 à 6 ans confirme la disponibilité de la construction, comme on le vérifiera. Ces observations nous conduisent à formuler une hypothèse didactique qui s'appuie sur les notions conjuguées de fait de langue, grammaire intuitive et besoin langagier.

Cette hypothèse est que l'on pourrait, dans le cadre de l'enseignement de la langue, renforcer par étapes la maitrise des tours pronominaux en allant d'un entrainement oral spécifique (lors d'une activité de langue "décrochée ", et ce dès la classe de grande section de maternelle, à partir des tours usuels et familiers et évidemment sans aucun recours terminologique) à une " explicitation grammaticale » (la grammaire " seconde » [Blanche-Benveniste, 1990, p.207-211]) qui se fasse ultérieurement (fin de l'école élémentaire, classes de collège et de lycée) et qui tende, progressivement, à tester les mécanismes d'une construction pronominale pour conduire les élèves à des emplois dont auparavant ils n'étaient pas eux-mêmes les "producteurs ». Un tel enseignement doit 
statuer sur les faits de langue à faire acquérir (le besoin langagier et les usages) avant de débattre des questions de terminologie ou d'analyse grammaticale.

\section{Les tours pronominaux, un indice de la maitrise linguistique?}

17 Nous avons retenu les tours pronominaux pour leur aptitude à interagir avec le cotexte de leur apparition et à mobiliser plusieurs questions, syntaxiques telles que la transitivité et la transformation passive, morphologiques telles que le choix de l'auxiliaire et la variation du pronom sujet, ou sémantiques telles que les types de procès, les données aspectuelles et la polysémie lexicale. Nous essayerons de procéder à l'examen fonctionnel de ces propriétés en nous attachant de façon complémentaire à identifier les besoins langagiers des locuteurs étant donné tel épisode raconté (le contexte), les réalisations effectives, les formes non disponibles (ou absentes) et les erreurs commises quand il s'en présente.

Du point de vue des références linguistiques, précisons ici que nous avons été confortée dans notre choix par la filiation de recherche des travaux de J. Stefanini, en diachronie (1962) et selon une approche guillaumienne des rapports forme-sens d'une voix pronominale (1992), jusqu'à - ce qui constitue la référence majeure - la recherche de C. Blanche-Benveniste et al. (1984, p.127-142) qui décrit, dans le cadre de l'approche pronominale, «les formes en se " telles qu'elles sont couramment réalisées en français parlé (1990, p. 52-53). La voix et l'aspect du verbe nécessitent par ailleurs un cadre conceptuel «systématique » qui permette d'appréhender «le temps» du procès (on complètera par Vassant, 1980a, 1980b ; Moignet, 1981 ; Melis, 1990 ; Barceló \& Bres, 2006), tandis que la productivité et la variété des tours contemporains requièrent un cadre d'analyse syntaxique homogène et opératoire tel que celui de C.Blanche-Benveniste (2010). La classification syntaxique opérée par J.-P. Boons, A. Guillet et C. Leclère (1976, p.120-163), plus tard augmentée du paramètre sémantique (Boons, 1987), constituera également un éclairage utile. Les tours pronominaux réunissent des propriétés hétérogènes qu'il convient de concevoir mais dont la profusion théorique dont ils sont l'objet, complique l'appréhension synthétique requise à des fins didactiques.

Nous avons sélectionné ci-dessous quatre emplois types, remarquables pour leur fréquence d'une séance à l'autre, leur fonction dans le récit, et/ou leur construction. Il s'agit respectivement des tours essentiellement pronominaux (s'absenter), d'un verbe de mouvement (se retourner), d'un verbe de parole (se dire) et de la structure causative (se faire avoir). Notre attention s'est portée sur les formes erronées (par exemple, elle le fâche, au lieu de elle se fâche, S1-55), quand elles nous permettent d'identifier un objet d'apprentissage.

\section{Verbes intrinsèquement pronominaux, s'absenter et s'enfuir}

Les deux verbes s'absenter (tableau 1) et s'enfuir (tableau 2) sont intrinsèquement pronominaux, c'est-à-dire qu'ils n'existent que sous cette forme, comme une soixantaine d'autres (Boons, Guillet \& Leclère, 1976, p. 159-163). Voici deux exemples d'occurrences de s'absenter. 


\begin{tabular}{|l|l|l|l|}
\hline Séances & $\mathbf{N}^{\mathbf{o}}$ & Loc & Interventions \\
\hline 4 & 12 & Paulo & elle dit la maman lapereaux je dois m'absenter pour la journée [...] \\
\hline 5 & 4 & Emilie & $\begin{array}{l}\text { que la maman doit s'absenter pour la journée et les petits lapins eh ben ils } \\
\text { doivent rester à la maison// }\end{array}$ \\
\hline
\end{tabular}

21 S'absenter apparait sous la forme de l'infinitif précédé du verbe modal devoir qu'il a dans le texte source. Seule change la personne : je dois ou elle doit, accompagné des variations de pronom réfléchi, je dois m'absenter, elle doit s'absenter. S'absenter apparait dans 8 séances sur 12 (au total, 13 occurrences dont aucune n'est déviante), ses formes se répartissent également entre les première et troisième personnes (l'alternance est présente dans la séance 6 : au tour 4 à la première personne, puis au tour 90 à la troisième). La base lexicale connue (absent > devoir être absente > devoir s'absenter) et la covariation morphologique ne posent pas de problème particulier pour le réemploi de ce verbe par les élèves. Notons toutefois qu'il n'apparait qu'une seule fois sous une forme fléchie, à l'imparfait (S11-20, et hum la maman elle a dit à ses quatre lapereaux qu'elle s'absentait pour la journée).

Quand dans une séance s'absenter n'a aucune occurrence, on peut l'expliquer de deux façons : i) la restitution des élèves en vient directement aux recommandations de la mère aux lapins, sans revenir sur le motif (S3-24 : elle a dit qu'ils pouvaient aller dans le pré mais pas dans le jardin de monsieur Grégory) ; ii) la séance commence in media res si l'on veut, et le rappel est aléatoire, conduit sans chronologie (par exemple, dans S7, dès le tour 2: monsieur Grégory il a dit à Pierre je vais te transformer en pâté...).

L'autre verbe intrinsèquement pronominal, s'enfuir (tableau 2), offre une variété supérieure d'emplois dont certains sont erronés. Tous (sauf un, $9: 4$; il s'est enfui) sont réalisés dans la séance 2 :

Tableau 2. S'enfuir

\begin{tabular}{|l|l|l|l|}
\hline Séance & $\mathbf{N}^{\mathbf{0}}$ & Loc & Interventions \\
\hline 2 & 68 & Sandra & il s'enfuit partout [...] \\
\hline 2 & 77 & Alice & $\begin{array}{l}\text { il est obligé d'enlever son gilet et de s'enfuir à toute vitesse et puis il se } \\
\text { cache dans un arrosoir }\end{array}$ \\
\hline 2 & 274 & Sandra & $\begin{array}{l}\text { non attends/ il voulait que attends/ il voulait qu'il s'enfuit et laisse ses } \\
\text { légumes tranquilles/ }\end{array}$ \\
\hline 2 & 317 & Elise & il s'est enfuyé \\
\hline
\end{tabular}

Les erreurs sont plutôt sémantiques. La première est occasionnée par la collocation de s'enfuir et de partout qui n'est pas juste : on peut courir partout (« dans tous les sens, toutes les directions») mais moins s'enfuir partout. Pourquoi? Peut-être parce que les deux 
verbes n'ont pas, lexicalement, la même valeur aspectuelle. On dira que s'enfuir comporte du fait de en (amalgamé à la base fuir, contrairement à s'en aller) une polarité initiale, également sensible dans fuir : "fuir de quelque part, de là $[=e n]$ ». Le danger-source est réalisé (en) contrairement à la destination du déplacement. Un phénomène comparable s'observe avec s'en aller: aller à la gare, dans les magasins, mais moins ?s'en aller à la gare. S'enfuir suppose donc un emploi intransitif que vient enfreindre le locatif partout. On comprend bien l'intention du locuteur : s'enfuir partout équivaut à s'enfuir n'importe où et vient appuyer l'idée d'une course "désordonnée» («dans tous les sens»). Courir qui, inversement, ne présente pas de polarité initiale, entre dans la catégorie des verbes de mouvement à polarité dite "centrée » (Boons, 1987) et c'est peut-être ce qui explique sa compatibilité avec partout, dans tous les sens.

La deuxième erreur sémantique (S2-274) relève aussi d'une confusion entre deux verbes synonymes: il voulait qu'il s'enfuie au lieu de il voulait qu'il parte, qu'il quitte son potager. L'insuffisance analytique de la mémoire sémantique du locuteur est remarquable ici : il y a en quelque sorte collusion syncrétique de deux points de vue. L'intention de Monsieur Grégory (qui veut le départ du lapin) contredit l'agentivité du procès de la fuite (le lapin s'enfuit, compte tenu des risques auxquels il réagit comme il peut). On complètera la discussion en remarquant que le verbe s'enfuir parait peu compatible avec un emploi à l'impératif (??Enfuis-toi), auquel l'usage préfère sauve-toi (ou, mieux encore, va-t'en), ce dont témoigne d'ailleurs le conseil donné au lapin par les oiseaux et dont se souviennent les élèves (S6-16 : ils disent vite vite sauve-toi ; $\mathrm{S} 8-52$ : il va pas arriver à se sauver).

Quant à l'erreur morphologique du participe passé, elle est assez banale (par analogie avec le participe passé des verbes en -er : *enfuyé au lieu de enfui). Elle nous intéresse cependant dans la mesure où elle apporte l'illustration qu'il est utile de faire travailler, oralement et couramment, les formes de participe de manière à ce que la finale vocalique soit acquise. Cela ne concerne pas seulement les classes de maternelle.

\section{Verbes de mouvement : se retourner (vers lui)}

27 Le verbe de mouvement se retourner, après le déplacement de s'enfuir, est également un indicateur intéressant des productions des élèves. Il est fréquemment utilisé mais il n'est pas sans poser une série de problèmes. Nous en énumérons quatre: l'opposition aspectuelle, la relation de causalité rappelée ou non entre l'éternuement du lapin et le mouvement de se retourner du jardinier, la suite adverbiale de ce verbe intransitif et la polysémie dérivationnelle de sa base lexicale tour.

Voici tous les emplois de se retourner au présent (tableau 3), puis au passé composé (tableau 4). Nous y avons associé une occurrence de se tourner (S3-233) et une autre de retourner non pronominal (\$1-39: il a retourné la terre).

Tableau 3. Se retourner au présent

\begin{tabular}{|l|l|l|l|}
\hline Séances & $\mathbf{N}^{\mathbf{0}}$ & Loc & Interventions \\
\hline 3 & 242 & Malo & et après/ monsieur Grégory il se retourne direct \\
\hline 4 & 83 & Paulo & monsieur Grégory se retourne vers lui avec un panier pour l'attraper \\
\hline
\end{tabular}




\begin{tabular}{|l|l|l|l|}
\hline 5 & 56 & Marco & $\begin{array}{l}\text { peut-être la mare elle était congelée du poisson/ et après il va dans la } \\
\text { cabane et il fait atchoum dans l'arrosoir/ et après et après monsieur } \\
\text { Grégory il se retourne/ et après [...] }\end{array}$ \\
\hline
\end{tabular}

Tableau 4. Se retourner au passé composé

\begin{tabular}{|c|c|c|c|}
\hline Séances & $\mathbf{N}^{\circ}$ & Loc & Interventions \\
\hline 1 & 10 & Shona & $\begin{array}{l}{[\ldots . .] \text { monsieur Grégory s'est retourné/ il a entendu atchoum derrière lui }} \\
{[\ldots . .]}\end{array}$ \\
\hline 1 & 35 & Sohaib & $\begin{array}{l}\text { il saute dans l'arrosoir et c'était vraiment pas une bonne idée parce que } \\
\text { c'était de l'eau glacée/ il a fait a/ atch/ atchoum/ et monsieur Grégory/il } \\
\text { s'est retourné et après il a couru si vite }\end{array}$ \\
\hline 1 & 36 & Mourad & $\begin{array}{l}\text { monsieur Grégory il sait qu'il était dans l'arrosoir parce qu'il a } \\
\text { éternué/ parce que il était glacé/ Pierre/ et il s'est retourné monsieur } \\
\text { Grégory }\end{array}$ \\
\hline 1 & 39 & Sihène & $\begin{array}{l}\text { eh bien quand Pierre est dans l'arrosoir/ eh bien il est/ il a encore } \\
\text { couru parce que monsieur Grégory il a retourné la terre et il l'a vu et Pierre } \\
\text { il a couru [...] }\end{array}$ \\
\hline 1 & 45 & Mourad & $\begin{array}{l}\text { et après Pierre quand il était glacé/ il a fait a/ il a fait a/ atchoum/ il a } \\
\text { éternué et monsieur Grégory il s'est fait/ il s'est fait entendu/ monsieur } \\
\text { Grégory/il s'est retourné derrière lui et il trouve le lapin et la maman elle } \\
\text { dit mais qu'est-ce qu'il fait mon fils/ pourquoi il y a pas ses habits/ dis } \\
\text { donc et il va avoir froid et après elle donne une tisane/ une tisane avec } \\
\text { quelque chose à manger et la mère et ses trois petits/ Pierre/ Romarin } \\
\text { il a peur/ et Pierre il dit rien à sa maman }\end{array}$ \\
\hline 3 & 233 & Melwan & et monsieur Grégory/il s'est tourné exactement pour l'attraper \\
\hline 9 & 26 & Sonia & $\begin{array}{l}\text { monsieur Grégory Pierre il a sauté dans l'arrosoir et monsieur Grégory } \\
\text { s'est vite retourné vers l'arrosoir }\end{array}$ \\
\hline 9 & 59 & Amélie & Pierre il a éternué si fort que monsieur Grégory il s'est retourné \\
\hline
\end{tabular}

Deux cas se présentent: une suite homogène de plusieurs verbes au présent ou une séquence complexe et hétérogène qui combine le passé composé au présent.

Le présent est appliqué à plusieurs verbes et la séquence narrative est homogène (S5-56) : il va..., il fait..., monsieur Grégory il se retourne. Les énoncés sont plutôt brefs et la succession des procès est ponctuée (et après, et après) et les envisage "globalement » (le procès y est vu en /+tension/ [Barcelò \& Bres, 2006]), sans mention de circonstances ou d'arrière-plan, ni codage d'une relation causale.

31 L'instruction aspectuelle n'est pas la même avec le passé composé où le procès est vu en / +extension/, c'est-à-dire au-delà de son effectuation, «à partir de la borne terminale atteinte du procès ». L'arrière plan de l'accompli que permet le passé composé est 
sensible quand l'intervention de l'élève (S1-35) amorce le rappel de la scène au présent de narration pour l'assortir ensuite d'un commentaire explicatif : il saute dans l'arrosoir et c'était vraiment pas une bonne idée parce que c'était de l'eau glacée/ il a fait a/ atch/ atchoum/ et monsieur Grégory/il s'est retourné et après il a couru si vite (...).

Le lien causal entre l'éternuement et le mouvement de Monsieur Grégory qui se retourne est marqué par la succession des énoncés, au présent (S5-56) ou au passé composé (S1-45). La relation est rarement codée comme elle l'est en S1-36 ou en S9-59.

L'expansion adverbiale de se retourner peut concerner la manière (la brusquerie du mouvement, sa rapidité : vite) ou l'orientation spatiale définie par un «but » (vers lui). À trois reprises, les suites adverbiales sont (plus ou moins) «erronées ». La première S3-242 - il se retourne direct, bien que familière, se révèle très expressive et adéquate, signifiant simultanément « tout à coup, brusquement » et la direction du mouvement qui atteint « directement » ce qui est " visé », à savoir le lapin. Quant à la seconde (S1-45), elle est passible de ce phénomène d'approximation ou de syncrétisme qui amalgame la cause (la source, la localisation du bruit: derrière lui) et le mouvement qui s'ensuit (se retourner). La troisième expansion (S3-233), il s'est tourné exactement pour l'attraper, revient également à faire coïncider le mouvement, le déclenchement avec une suite possible (attraper le lapin).

La polysémie de retourner apparait avec S1-39: Monsieur Grégory il a retourné la terre. L'énoncé traduit un contresens fondé sur la valeur d'emploi pronominal (se retourner) et le régime direct de l'emploi non pronominal (retourner la terre). Le verbe retourner comme beaucoup d'autres est accessoirement pronominal : le pronom réfléchi se est le support de l'action de retourner: Monsieur Grégory retourne « lui-même » en quelque sorte. Notons que le choix de la terre comme support d'action n'est pas tout à fait aléatoire dans la mesure où il a un rapport avec l'action de "jardiner » et où l'expression locutionnelle retourner ciel et terre (pour retrouver quelqu'un ou quelque chose) est peut-être connue du locuteur.

\section{Verbes de parole : dire et se dire, demander et se demander}

Les verbes de parole ou de communication, dire et demander, ne sont évidemment pas toujours pronominaux. Le tour pronominal signale un procès de parole qu'on s'adresse à soi-même : se demander, se dire, et le pronom réfléchi est doté d'une fonction de datif. Cette valeur est-elle perçue par les élèves qui ne recourent pas au tour pronominal (par exemple en S3-277 : et sa maman elle dit pourquoi il a perdu toutes ses affaires)? Voici quelques-unes des occurrences de (se) dire et se demander, choisies pour la variation des usages de discours rapporté, alors qu'ils sont tous reliés au même propos (pourquoi il a perdu ses vêtements).

Tableau 5. Se dire, se demander

\begin{tabular}{|l|l|l|l|}
\hline Séances & $\mathbf{N}^{\mathbf{0}}$ & Loc & Interventions \\
\hline 4 & 154 & Judicaël & pourquoi il a encore perdu ses vêtements elle se disait \\
\hline 4 & 155 & Mélissa & elle se dit pourquoi il a perdu ses vêtements \\
\hline
\end{tabular}




\begin{tabular}{|l|l|l|l|}
\hline 4 & 175 & Siméon & $\begin{array}{l}\text { on croit qu'il est re-allé dans le potager de monsieur Grégory parce que } \\
\text { la maman de Pierre elle se demandait pourquoi il avait encore perdu ses } \\
\text { vêtements ça fait deux fois cette semaine }\end{array}$ \\
\hline 3 & 277 & Melwan & et sa maman elle dit pourquoi il a perdu toutes ses affaires \\
\hline 6 & 29 & Emilie & $\begin{array}{l}\text { et la maman elle dit sacré Pierre/ il a encore perdu son man son } \\
\text { manteau/ ses chaussures//je me demande qu'est-ce qu'il fait// }\end{array}$ \\
\hline
\end{tabular}

L'alternance du discours direct (S4-154, avec postposition de la phrase attributive, reformulée par le locuteur suivant, en S4-155, qui déplace la phrase attributive et change le temps du verbe) et du discours indirect (S6-29: je me demande qu'est-ce qu'il fait) est une caractéristique commune, non spécifique des verbes pronominaux; elle se pose quel que soit le verbe qui introduit le discours rapporté (par exemple S11-30, les oiseaux lui disent de se sauver).

On peut suggérer que l'opposition entre dire et se dire soit travaillée avec les élèves dans le cadre d'une activité décrochée, par exemple le mime d'une activité de parole adressée ( dire) et d'une autre, réflexive (se dire).

\section{Structure causative et médio-passive, la voix moyenne : se faire avoir (disputer, attraper, manger...)}

On est avec cette structure au cœur de la difficulté syntaxique des verbes pronominaux.

Se faire, mais aussi se laisser, se voir sont des auxiliaires de médio-passif et permettent de déplacer en position de sujet syntaxique le patient sémantique. Voici les exemples que nous avons relevés :

Tableau 6. Se faire + V

\begin{tabular}{|l|l|l|l|}
\hline Séances & $\mathbf{N}^{\mathbf{0}}$ & Loc & Interventions \\
\hline 1 & 45 & Mourad & $\begin{array}{l}\text { et après Pierre quand il était glacé/ il a fait a/ il a fait a/ atchoum/ il a } \\
\text { éternué et monsieur Grégory il s'est fait/ il s'est fait entendu [...] }\end{array}$ \\
\hline 2 & 311 & M & il est perturbé par ses émotions ça veut dire quoi \\
\hline 2 & 312 & Gaël & de sa journée/ il a failli se faire attraper \\
\hline 3 & 18 & Melwan & et puis son papa il reviendrait jamais parce qu'il s'est fait manger \\
\hline 3 & 26 & Melwan & parce que s'il y va/ il va se transformer en pâté \\
\hline 3 & 55 & Viviane & $\begin{array}{l}\text { il (le papa des lapins) a été dans le jardin de monsieur Grégory et il s'est } \\
\text { fait cuire par madame Grégory et monsieur Grégory il l' a mangé }\end{array}$ \\
\hline 3 & 149 & Melwan & il court tout vite \\
\hline 3 & 150 & M & pourquoi \\
\hline
\end{tabular}


\begin{tabular}{|l|l|l|l|}
\hline 3 & 151 & Viviane & parce que sinon/ il va se faire manger \\
\hline
\end{tabular}

En S1-45, l'erreur qui porte sur la forme du verbe - entendu au lieu de entendre - est peutêtre significative du sens passif que l'auxiliaire se faire construit. La permutation des rôles de la diathèse place le régime direct, interprétable comme patient du procès, en position de sujet du verbe. Un autre exemple est significatif de la fragilité du même phénomène (S3-26) : l'omission du verbe causatif faire, il va se transformer en pâté au lieu de il va se faire transformer. Dans ce dernier cas, l'ajout de l'agent par le jardinier aurait permis de discriminer les deux formulations : ${ }^{*} i l$ va se transformer en pâté par le jardinier; il va se faire transformer en pâté par le jardinier.

Dans tous les cas recensés, le sujet patient de ces constructions est soit le père des lapins soit Pierre le lapin. L'idée commune est souvent que Pierre risque de ou a failli subir le même sort que son père (être mangé). Rappelons que le texte source mentionne que le père a été transformé en civet par madame Grégory, ce que S3-55 formule en disant s'est fait cuire. La solution est discutable mais lui préférera-t-on s'est fait manger? Ce n'est pas sûr.

Comparons :

(6a)

Il s'est fait cuire

Il s'est fait manger

Il s'est fait attraper (au sens de se faire gronder)

Il apparait que, débarrassés de leur environnement, les tours prennent tous les trois un sens métaphorique et familier : se faire cuire au soleil, se faire manger par l'adversaire, ou se faire gronder (houspiller, réprimander). En d'autres termes, la productivité du tour pronominal en se faire $+V_{\text {Infinitif }}$ présente un cas intéressant de réanalyse (Béguelin, Corminboeuf \& Johnsen, 2014), où la réalisation des locuteurs est à la frontière d'un tour lexicalisé et d'une construction libre. Ici, l'interprétation du tour produit qui est sans ambigüité celle d'une construction libre (se faire + manger, cuire, attraper) et la connaissance d'usages lexicalisés (se faire avoir ou se faire disputer comme exemples types) qui sont adéquats à la situation décrite, favorisent l'apparition des expressions relevées, à la frontière de deux types de réalisations figées et libres. Dans cette hypothèse, les exemples (8a) attestent d'une réalisation de "français avancé ", non standard, mais que l'on aurait du mal à assimiler strictement à des « fautes ». Un cas comme celui-ci confirme s'il en était besoin l'importance de travailler avec les élèves, et au-delà de la seule école maternelle, sur les faits de langue eux-mêmes bien avant d'en analyser les structures proprement dites. Rappelons à cet égard que la structure causative en se faire est d'une grande complexité sémantique et syntaxique. Le patient du procès y occupe une place de sujet-objet, grâce au pronom réfléchi (il se) et l'agent peut être absent (par...). Une telle structure demande par ailleurs un examen attentif des clitiques et des accords orthographiques (elle se l'est fait voler; et non sa voiture elle se l'est *faite voler).

Quel bilan tirer des observations qui précèdent? Le classement opéré, après le premier critère de la forme pronominale des verbes, a dégagé des formes types (s'absenter, se retourner, se dire, se faire manger) sur des bases qui sont sémantiques et lexicales. D'après les emplois attestés dans le corpus, il semblerait que les locuteurs, motivés par leur compréhension du texte, recourent à leur mémoire (lexicale). Ils connaissent probablement, de façon intuitive, la construction non pronominale et alternative des 
verbes dire et retourner. Et, comme on l'a déjà suggéré plus haut, on pourrait concevoir une activité de langue "décrochée " qui explicite les deux formes: dire qc/se dire, retourner qc/se retourner, en élargissant la série à des verbes comme sauver qn/se sauver, appeler qn/s'appeler, ou reposer qc/se reposer. Nous reviendrons sur ces propositions dans la partie suivante, après l'examen de l'épisode du groseillier.

\section{Le groseillier, un épisode complexe aux restitutions contrastées}

\section{Complexité de l'énoncé source qui constitue le centre de l'épisode « groseillier»}

Rappelons le discours source qui restitue l'épisode du groseillier :

« Il court ventre à terre aussi vite qu'il le peut. Il est essoufflé, épuisé. Mais c'est alors que le bouton de son gilet se prend dans le filet qui recouvre le plant de groseilles qu'il est en train de traverser. Catastrophe! Il regarde autour de lui, les oiseaux qui l'observent lui crient de se sauver! Monsieur Grégory arrive, un panier à la main, prêt à l'attraper. Vite, vite, Pierre ! OUF ! Juste à temps ! Pierre vient d'enlever son gilet! Il reprend sa course folle, passant juste sous les jambes de monsieur Grégory ».

On peut décomposer l'épisode en quatre temps, repris dans le tableau (tableau 7, infra) :

- la fuite interrompu par l'épisode du groseillier

- le bouton accroché

- l'abandon du gilet

- la reprise de la fuite (rendue possible par l'abandon du gilet)

Le texte source présente l'épisode selon deux modalités distinctes: l'assertion d'un énoncé long, complexe en raison du clivage (c'est alors que...) et remarquable pour deux syntagmes nominaux (le bouton de son gilet; le plant de groseilles qu'il est en train de traverser) focalisés tous les deux sur Pierre. La deuxième modalité, exclamative, introduit du discours direct libre (Vite, vite, Pierre! Ouf! Juste à temps!) qu'elle segmente, « imitant » la précipitation de la décision du personnage et sa fuite «de justesse». L'intonation à la lecture a dû restituer cet effet de suspens que l'écrit symbolise par quatre points d'exclamation ponctuant quatre clauses indépendantes dont la dernière seule est assertive et renoue avec le fil du récit (mais sur le mode de l'analepse ${ }^{6}$ : Pierre vient d'enlever son gilet). La complexité référentielle de la scène se double donc d'une certaine complexité stylistique qui use des ressources propres à l'écrit (longueur de l'énoncé, densité lexicale, variété modale et polyphonie).

\section{Recensement de l'épisode dans les 12 séances du corpus CLEA}

Dans le tableau suivant (tableau 7), nous avons recueilli les formulations de l'épisode du groseillier telles qu'elles ont été réalisées dans les séances et selon une décomposition de l'épisode en quatre phases (1. l'obstacle du filet, 2. l'incident du bouton coincé, 3. le gilet abandonné et 4 . la course reprise). Sous le numéro des séances, dans la première colonne du tableau, figure entre parenthèses le nombre de locuteurs (loc.) différents qui contribuent à la restitution de l'épisode. Souvent, par exemple pour la séance 1, nous 
avons fusionné les cellules du filet et du bouton accroché ( $2^{\mathrm{e}}$ et $3^{\mathrm{e}}$ colonnes) quand les phases 1 et 2 sont associées, sous la forme d'un locatif (s'accrocher à, dans, sur, + locatif).

Tableau 7. Les 4 phases de l'épisode du groseillier

\begin{tabular}{|c|c|c|c|c|}
\hline & Filet à groseilles & Bouton accroché & Gilet abandonné & Course reprise \\
\hline $\begin{array}{l}1 \\
(2 \\
\text { loc. })\end{array}$ & \multicolumn{2}{|c|}{$\begin{array}{l}\text { (10) il s'est accroché dans un légume } \\
\text { (69) La chemise était accrochée aux pommes } \\
\text { de terre }\end{array}$} & $\begin{array}{l}\text { (10) et après au moment où } \\
\text { il fallait il enlève son gilet } \\
(69) \text { et après il a enlevé sa } \\
\text { chemise }\end{array}$ & $\begin{array}{l}\text { (69) et après il a } \\
\text { recouru }\end{array}$ \\
\hline $\begin{array}{l}2 \\
(3 \\
\text { loc. })\end{array}$ & & (70) il coince son gilet & $\begin{array}{l}\text { (74) il essaie d'enlever son } \\
\text { gilet } \\
\text { (76) il arrivait pas à enlever } \\
\text { son gilet } \\
\text { (77) il est obligé d'enlever } \\
\text { son gilet }\end{array}$ & $\begin{array}{l}\text { (77) et de s'enfuir à } \\
\text { toute vitesse }\end{array}$ \\
\hline $\begin{array}{l}3 \\
(4 \\
\text { loc. })\end{array}$ & \multicolumn{2}{|c|}{$\begin{array}{l}\text { (188) il était accroché sur le fil/ le bouton } \\
\text { (193) il s'est accroché au filet }\end{array}$} & $\begin{array}{l}\text { (206) il essaie d'enlever dans } \\
\text { le filet }\end{array}$ & $\varnothing$ \\
\hline $\begin{array}{l}4 \\
(5 \\
\text { loc. })\end{array}$ & \multicolumn{2}{|c|}{$\begin{array}{l}\text { (45) et son gilet il est accroché à la branche } \\
\text { (50) au filet des groseilles } \\
\text { (53) son gilet s'attache dans les groseilles }\end{array}$} & (55) et il enlève vite son gilet & $\begin{array}{l}\text { (55) et après il } \\
\text { reprend sa course } \\
\text { folle }\end{array}$ \\
\hline $\begin{array}{l}5 \\
(4 \\
\text { loc. })\end{array}$ & $\begin{array}{l}\text { (5) il était coincé } \\
\text { dans le filet à } \\
\text { groseilles }\end{array}$ & $\begin{array}{l}\text { (19) il y a un bouton qui } \\
\text { est accroché/ le bouton } \\
\text { de son gilet à Pierre }\end{array}$ & $\begin{array}{l}\text { (20) Pierre eh ben juste à } \\
\text { temps il trouve une solution } \\
\text { (22) il enlève son manteau } \\
\text { (70) et son gilet/ il a perdu } \\
\text { son gilet/ il était coincé alors } \\
\text { il a enlevé son gilet }\end{array}$ & $\begin{array}{l}\text { (70) et il a rattrapé } \\
\text { sa course }\end{array}$ \\
\hline $\begin{array}{l}6 \\
(3 \\
\text { loc. })\end{array}$ & \multicolumn{2}{|c|}{$\begin{array}{l}\text { (8) il s'est pris les fils des groseilles } \\
\text { (15) il s'a accroché son bouton de gilet dans } \\
\text { les plants de groseilles }\end{array}$} & (17) il enlève sa veste & $\varnothing$ \\
\hline 7 & \multicolumn{4}{|l|}{$\varnothing$} \\
\hline $\begin{array}{l}8 \\
(3 \\
\text { loc. })\end{array}$ & \multicolumn{2}{|c|}{$\begin{array}{l}\text { (52) son bouton il s'est attaché à un fil où se } \\
\text { trouvent euh comment ça s'appelle (53) les } \\
\text { cerises } \\
\text { (61) et son bouton il s'est accroché au filet } \\
\text { (66) et aussi son bouton comme il s'est pris } \\
\text { au filet }\end{array}$} & $\begin{array}{l}\text { (68) eh ben il a enlevé son } \\
\text { gilet parce qu'il était cassé } \\
\text { maintenant/ } \\
\text { (77) et quand il était } \\
\text { accroché il arrivait plus à se } \\
\text { sauver et juste à temps il a } \\
\text { enlevé son gilet }\end{array}$ & $\begin{array}{l}\text { (68) et il l'a enlevé } \\
\text { pour reprendre la/ } \\
\text { pour courir plus } \\
\text { vite }\end{array}$ \\
\hline
\end{tabular}




\begin{tabular}{|c|c|c|c|c|}
\hline $\begin{array}{l}9 \\
(4 \\
\text { loc. })\end{array}$ & $\begin{array}{l}(10) \quad \text { monsieur } \\
\text { Grégory l'avait un } \\
\text { filet }\end{array}$ & $\begin{array}{l}\text { (11) et Pierre son } \\
\text { bouton était fait } \\
\text { attraper au filet } \\
\text { (28) il s'est attrapé par } \\
\text { le bouton parce qu'il } \\
\text { était dans le filet } \\
\text { (112) son bouton/ le } \\
\text { bouton de Pierre de son } \\
\text { gilet vient de s'attraper } \\
\text { parle fil }\end{array}$ & (109) il a enlevé son gilet & $\varnothing$ \\
\hline $\begin{array}{l}10 \\
(2 \\
\text { loc. })\end{array}$ & $\begin{array}{l}\text { (46) il s'est pris le } \\
\text { filet de ses } \\
\text { groseilles }\end{array}$ & (22) il était accroché & $\begin{array}{l}\text { (46) et puis après et après il } \\
\text { a enlevé vite son gilet }\end{array}$ & $\varnothing$ \\
\hline $\begin{array}{l}11 \\
(2 \\
\text { loc. })\end{array}$ & \multicolumn{2}{|c|}{$\begin{array}{l}\text { (30) eh ben tout à coup le fil/ le bouton de } \\
\text { son gilet s'est pris dans le plant de groseilles }\end{array}$} & $\begin{array}{l}\text { (28) il a enlevé son gilet } \\
\text { (30) ouf juste à temps Pierre } \\
\text { arrive à enlever son gilet }\end{array}$ & $\begin{array}{l}\text { (28) et il reprend sa } \\
\text { course folle }\end{array}$ \\
\hline $\begin{array}{l}12 \\
(2 \\
\text { loc. })\end{array}$ & \multicolumn{2}{|c|}{$\begin{array}{l}\text { (22) il était accroché son bouton/ il était } \\
\text { accroché sur le filet }\end{array}$} & $\begin{array}{l}\text { (18) il a enlevé sa veste et il } \\
\text { s'est détaché du filet } \\
\text { (22) après il a enlevé son } \\
\text { gilet }\end{array}$ & $\varnothing$ \\
\hline
\end{tabular}

Le tableau qu'on vient de lire synthétise l'épisode du groseillier tel qu'il est rapporté par les élèves, et dont nous avons par ailleurs rapporté "linéairement» les extraits en annexe. Le nombre de locuteurs différents est variable, cela va de 2 (S1, S10, S11 et S12) à 5 (S4). Il faudrait confronter ce paramètre avec la distance plus ou moins grande qui sépare les tours de parole traitant de l'épisode du groseillier. C'est ainsi que la variété de locuteurs, dans la séance 4 , va de pair avec la proximité des tours de parole $(45,50,53$, 55), ce qui semblerait signifier une posture collaborative non négligeable dans l'élaboration de l'épisode. La collaboration donne lieu, de proche en proche, à des reformulations du locatif (nous soulignons).

Tableau 8. La variété des locuteurs de la séance 4

\begin{tabular}{|l|l|l|l|}
\hline Séance & No & Loc & Interventions \\
\hline 4 & 45 & Keliane & et son gilet il est accra accroché au/ à la branche \\
\hline 4 & 50 & Thiébaud & au filet des groseilles \\
\hline 4 & 53 & Keliane & son gilet s'attache/ dans les groseilles \\
\hline
\end{tabular}

Plus précisément, le tableau 7 permet de relever un contraste "lexique-grammaire » entre les séances 8 et 9 concernant la phase du «bouton accroché ». En S8, deux locuteurs, Viviane et Sergueï, procèdent à une investigation lexicale. À partir d'une structure syntaxique identique (se $V \grave{a}$ ), ils opèrent une recherche lexicale : s'attacher $\grave{a}$ 
(52), s'accrocher à (61), se prendre à (66). La variation porte sur l'unité verbale. Inversement, l'extrait de la séance 9 répertorié dans le tableau 7 procède au contraire à une investigation syntaxique. Les unités lexicales sont identiques - le bouton, s'attraper, le fil (filet) - mais les constructions essayées ne sont pas les mêmes: son bouton était fait attraper (11), s'est attrapé par le bouton (28), le bouton vient de s'attraper par le fil (112). La distance relative entre ces différents essais syntaxiques de S9 joue son rôle et l'on peut se demander si la variation lexicale ( $\mathrm{S} 8$ ) opère mieux dans des interventions rapprochées, contre une mémorisation à distance des unités lexicales (son bouton + s'attraper, S9), pour lesquelles la forme syntaxique n'est pas stabilisée. Nous consacrons le point suivant à ce point particulier, qui constitue l'une des difficultés linguistiques majeures, combinant lexique et syntaxe.

Enfin, le tableau 7 permet de remarquer que la dernière phase de la course reprise (la quatrième colonne du tableau) est absente des séances $3,5,6,9,10$ et 12 (l'absence est notée $\varnothing$ dans les cellules).

Trois cas se présentent :

- La course folle est associée à la sortie de l'arrosoir d'eau glacée. Par exemple (S6-20) : et après il va dans l'arrosoir et l'eau il était tellement tellement gelée qu'il a fait atchoum et après monsieur Grégory il se retourne et après il s'approche de l'arrosoir et après il reprend cette course folle et après il a trouvé la porte.

- La fuite de Pierre constitue le conseil que lui adressent les oiseaux. Par exemple, (S9-18) : et les oiseaux il a dit/ cours cours cours vite Pierre.

- La course folle précède l'épisode du groseillier, et non l'inverse. Par exemple (S8-61) : et son bouton il s'est accroché au au filet quand il courait ventre à terre.

Ces observations semblent indiquer que l'évènement de premier plan, la fuite du lapin, se réalise « en continu » à des emplacements indifférenciés, s'effaçant au profit de notations singulières qui caractérisent une péripétie et son décor. La décomposition du procès filet/ bouton/gilet comporte sa représentation chronologique contrairement à la fuite uniforme, qui est reprise, interrompue, reprise...

Le tableau 7 montre que la réussite de la restitution de l'épisode dans ses quatre phases va de pair, en $\mathrm{S} 5$, avec la variété des locuteurs et une répartition significative des interventions $(5,19-20-22,70)$.

Le gilet pris dans le groseillier constitue un obstacle à l'avancée du lapin, lequel obstacle est dramatique et marquant (58-77 : et quand il était accroché il arrivait plus à se sauver et juste à temps il a enlevé son gilet), et il est mémorisé sous la forme des deux procès successifs s'accrocher/être accroché au filet et enlever son gilet. Nous consacrons la partie suivante à l'examen du procès «s'accrocher ». Le verbe est d'autant plus remarquable qu'il n'est pas utilisé dans le texte source (où l'on trouve se prendre et enlever).

\section{Phases de procès et constructions verbales : s'accrocher ou être accroché : représentation syncrétique, approximative et/ou « reformulation lacunaire » (Martinot, 2000) ?}

Le tableau 7 qu'on vient de commenter, rappelle la variété lexicale des solutions, pronominales ou non, auxquelles recourent les élèves pour évoquer la scène du bouton du gilet: ( $\left.s^{\prime}\right)$ accrocher, coincer, attacher, (se) prendre, (se faire) attraper. Rappelons que le texte source dit que «le bouton de son gilet se prend dans le filet $[. .$.$] ». Chez les élèves, la$ 
solution majoritaire est était accroché, autrement dit le verbe accrocher au plus-que-parfait en emploi non pronominal. Un nouveau tableau (tableau 9), reprend ces emplois. Dans ce tableau, ci-dessous, quand plusieurs références figurent dans la même parenthèse, cela signifie que les tours de parole sont le fait d'un même locuteur ; par exemple en S3-188, 190, etc., sont réalisés par Viviane. Le gras signale la variété des solutions dans la colonne « occurrences » et la seule solution (s')accrocher dans la colonne « verbes ».

Tableau 9. (S')accrocher

\begin{tabular}{|c|c|c|}
\hline Séances & Occurrences & Verbes \\
\hline 1 & $\begin{array}{l}\text { (10) il s'est accroché dans un légume } \\
\text { (69) la chemise de Pierre elle était accrochée aux } \\
\text { pommes de terre }\end{array}$ & $\begin{array}{l}\text { S'ÊTRE ACCROCHÉ, PC } \\
\text { ÊTRE ACCROCHÉ, PQP }\end{array}$ \\
\hline 2 & (70) il coince son gilet & COINCER, Pr \\
\hline 3 & $\begin{array}{l}(188,190,191,210) \text { il était accroché sur le fil/ le } \\
\text { bouton }\end{array}$ & ÊTRE ACCROCHÉ, PQP \\
\hline 4 & $\begin{array}{l}\text { (45) son gilet il est accroché à la branche } \\
\text { (53) son gilet s'attache dans les groseilles }\end{array}$ & $\begin{array}{l}\text { ÊTRE ACCROCHÉ, PC } \\
\text { S'ATTACHER, Pr }\end{array}$ \\
\hline 5 & $\begin{array}{l}\text { (5) il était coincé dans le filet } \\
\text { (19) il y a un bouton qui est accroché } \\
\text { (70) il était coincé }\end{array}$ & $\begin{array}{l}\text { ÊTRE COINCÉ, PQP } \\
\text { ÊTRE ACCROCHÉ, PC } \\
\text { ÊTRE COINCÉ, PQP }\end{array}$ \\
\hline 6 & $\begin{array}{l}\text { (8) il s'est pris les fils de groseilles } \\
\text { (15) il s'a accroché son bouton }\end{array}$ & $\begin{array}{l}\text { S'ÊTRE PRIS, PC } \\
\text { S'ÊTRE } \\
\text { ACCROCHÉ, PC (S'AVOIR ?) }\end{array}$ \\
\hline 7 & / & \\
\hline 8 & $\begin{array}{l}(49,52,71) \text { son bouton il s'est attaché à un fil } \\
\text { (66) il s'est pris au filet } \\
\text { (77) il était accroché }\end{array}$ & $\begin{array}{l}\text { S'ÊTRE ATTACHÉ, PC } \\
\text { S'ÊTRE PRIS, PC } \\
\text { ÊTRE ACCROCHÉ, PQP }\end{array}$ \\
\hline 9 & $\begin{array}{l}\text { (10) son bouton était fait attraper } \\
\text { (28) il s'est attrapé par le bouton } \\
\text { (112) le bouton de Pierre de son gilet vient de s'attraper } \\
\text { par le fil }\end{array}$ & $\begin{array}{l}\text { ÊTRE FAIRE ATTRAPER, PQP } \\
\text { S'ÊTRE ATTRAPÉ, PC } \\
\text { VENIR DE S'ATTRAPER, Pr }\end{array}$ \\
\hline 10 & $\begin{array}{l}\text { (22) il était accroché } \\
\text { (46) il s'est pris le filet }\end{array}$ & $\begin{array}{l}\text { ÊTRE ACCROCHÉ, PQP } \\
\text { S'ÊTRE PRIS LE FILET, PC }\end{array}$ \\
\hline 11 & (30) le bouton s'est pris dans le plant... & S'ÊTRE PRIS, PC \\
\hline
\end{tabular}




\begin{tabular}{|l|l|l|}
\hline 12 & $\begin{array}{l}\text { (18) il s'est détaché du filet } \\
\text { (22) il était accroché son bouton } \\
\text { (39) il s'est pris dans le bouton }\end{array}$ & S'ÊTRE DÉTACHÉ, PC \\
ÊTRE ACCROCHÉ, PQP \\
S'ÊTRE PRIS, PC \\
\hline
\end{tabular}

Peut-on dire que la construction du verbe s'accrocher comporte à la fois un sens de «mouvement ", de « localisation » et/ou de «manière »? On peut convenir que le verbe lui-même est de polarité finale ou d'orientation résultative (contrairement par exemple à se détacher qui aura la propriété de préparer la suite de l'épisode), et que la notation de manière implique l'indication d'un "mouvement interrompu». Quant à l'information locative, elle ne réside pas dans le verbe lui-même mais dans l'une des valences régies (au filet, dans le plant...). Ce que l'on constate à travers la paraphrase « $\mathrm{X}$ est resté accroché au fil ». L'effet de sens qui domine les reformulations de s'accrocher est bien la conjonction de l'obstacle survenu (coincer) qui confère brutalement à l'agent (s'enfuir) le statut d'un patient impuissant (se faire attraper).

Le verbe accrocher non pronominal est d'usage courant: va accrocher ton gilet au portemanteau. C'est bien l'état résultant qui est donné : l'activité accrocher doit déboucher sur un prédicat statif être accroché. Quant au verbe pronominal s'accrocher il est d'usage spécifique (les gilets s'accrochent aux porte-manteaux), et il, a dans d'autres situations (scolaires ou familiales), des concurrents potentiels: se ranger, se mettre, s'attacher, se fermer (accroche ton gilet pour ferme, ou boutonne, ton gilet; un gilet ça se ferme; ou bien accroche ton gilet pour suspends ton gilet au porte-manteau).

Dans le cas présent, c'est le verbe support se prendre qui décrit la phase de l'obstacle survenu. La diathèse Patient-Agent ne recouvre pas l'opposition Animé-Inanimé ni celle $\mathrm{du}$ Sujet et de l'objet de la construction du verbe. Pour satisfaire une représentation sémantique de l'épisode raconté qui soit complète du point de vue de la causalité, il faut introduire un instrument qui a des propriétés locatives (le filet qui « retient » le lapin).

On constate (tableau 10) que se prendre ne connait que trois réalisations, dont la seconde est familière et la troisième fautive (l'objet construit comme un locatif : dans le bouton) :

Tableau 10. Se prendre

\begin{tabular}{|l|l|l|l|}
\hline Séances & $\mathbf{N}^{\mathbf{o}}$ & Loc & Interventions \\
\hline 8 & 77 & Sihame & $\begin{array}{l}\text { euh euh euh son bouton du gilet il a il était accroché/ et quand il était } \\
\text { gilet// }\end{array}$ \\
\hline 6 & 8 & Alexis & {$[\ldots .$.$] il s'est pris les les/fils de groseilles$} \\
\hline 12 & 39 & Jean & {$[\ldots .$.$] il s'est pris dans le bouton$} \\
\hline
\end{tabular}

61 Étant donné par ailleurs la fréquence du verbe prendre comme support de tours ordinaires (prendre le train, le courrier, le pain, ses affaires, etc.) qui sont couramment complétés par des locatifs (je te prendrai devant l'école), il faut faire l'hypothèse que se prendre est rendu moins accessible en raison précisément de son emploi pronominal. La reformulation 
dominante, est (était) accroché à, s'appuie sur le prédicat résultatif en être, que le sujet soit animé ou non, et lui adjoint un locatif, par exemple :

Tableau 11. S'accrocher à

\begin{tabular}{|l|l|l|l|}
\hline Séance & No & Loc & Intervention \\
\hline 4 & 45 & Keliane & et son gilet il est accra accroché au/ à la branche \\
\hline
\end{tabular}

Dans plusieurs exemples, cités sous (10, se prendre), les locatifs et la préposition qui les régit sont problématiques: dans un légume (S1-10), au fil (S1-33), aux pommes de terre (S1-69), ... sur les pommes de terre (S1-69), etc. Les solutions retenues privilégient une interprétation « locative » (quelque part), plutôt que «agentive » (« être retenu par») et « processuelle» (s'accrocher à).

63 Est assez frappante enfin la variété lexicale des verbes accrocher, attacher, coincer et attraper, sans parler de l'antonyme se détacher qui marque la polarité initiale du procès suivant (la phase 3 de l'épisode "groseillier », illustré en S12-18). On peut y voir la trace d'une surgénéralisation dont il résulte une approximation via les synonymes qui est sur le moment jugée suffisante, mais qui perd la spécificité sémantique de chaque lexème, que des collocations permettraient d'activer: attacher ses cheveux, coincer une feuille de papier, attraper un rhume ou détacher un timbre. Tous ces exemples satisferaient une transformation pronominale: les cheveux s'attachent..., une feuille s'est coincée, les rhumes s'attrapent..., un timbre se détache... La spécificité lexicale des constructions verbales va de pair avec la permutation des rôles syntaxiques et correspond au «se de formulation » de C. Blanche-Benveniste et al. (1984, p. 130-133).

L'épisode du groseillier confirme ce que nous avions déjà entrevu avec l'auxiliaire se faire : l'intrication des composants sémantiques et syntaxiques dans des constructions complexes et l'intérêt didactique qu'il y aurait à entrainer les élèves à leur "analyse ». Nous entendons par là un entrainement à produire des transformations simples dans des réalisations binaires et équivalentes, telles que nous les indiquons dans le point suivant.

\section{Des activités décrochées sur les tours pronominaux?}

On peut esquisser des propositions d'activités en langue, qui soient "décrochées » et complètent les séances de langage. Leur objet consiste en un entrainement limité dans le temps et fondé sur la reprise orale de quelques énoncés standards. Par exemple, les suivants :

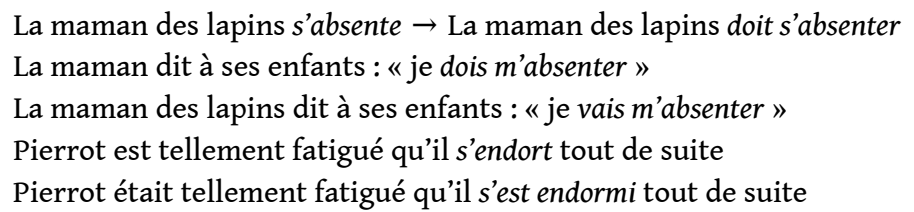

On pourrait par ailleurs esquisser implicitement dans ces reprises orales le principe d'une transformation régulière. C'est ce que nous montrons ci-dessous avec les formes fléchies de l'impératif. L'objectif est d'associer l'infinitif pronominal (ci-dessous, entre parenthèses) à une forme fléchie, en activant ou non à cette étape d'entrainement la variation du pronom: 
(Se sauver) Les oiseaux lui crient de se sauver

(Se sauver) Il faut te sauver $\rightarrow$ Sauve-toi

(Se méfier) il faut se méfier $\rightarrow$ Méfie-toi

(Se méfier) Il faut te méfier de Monsieur Grégory $\rightarrow$ Méfie-toi de Monsieur Grégory

(S'arrêter de courir et se reposer) Il faut t'arrêter de courir et te reposer $\rightarrow$ Arrête-toi de

courir et repose-toi

Ces suggestions ont surtout valeur d'illustration et méritent d'être encore discutées.

L'hypothèse qui les sous-tend est la suivante: les verbes pronominaux réalisés sont activés par les élèves sur des bases sémantiques et lexicales; il conviendrait de motiver également, à l'aide des reprises indiquées, la variation structurelle et syntaxique des verbes pronominaux.

C'est ainsi que l'on pourrait enfin ébaucher la commutation des clitiques à travers des reprises telles que :

(Fâcher quelqu'un, le (la) fâcher, se fâcher contre lui, elle)

Il a peur de fâcher sa maman $\rightarrow$ Il a peur de la fâcher $\rightarrow$ Il a peur qu' elle se fâche

contre lui

Il fâche sa maman $\rightarrow$ il la fâche $\rightarrow$ Elle se fâche contre lui

(Cacher quelque chose, se cacher)

Il cache dans son dos le gilet déchiré $\rightarrow$ il le cache dans son dos

Il se cache dans l'arrosoir

69 Avec ces entrainements, l'objectif est de rendre sensible la " polysémie syntaxique » des verbes retenus, dès lors qu'un même verbe peut régir un inanimé (gilet) ou un animé (se cacher soi-même).

Ces entrainements, conçus comme des « activités décrochées », n'ont - répétons-le - que valeur d'exemple. Il s'agit d'accompagner une séance de langage collective qui a pour objet la restitution d'un récit, par une séance de langue qui sélectionne des tours dans le but de les faire entendre, reconnaitre, répéter, mémoriser puis réinvestir (transférer) dans une nouvelle circonstance), à l'instar de ce qui se fait en conjugaison. On a voulu rappeler ainsi que les transformations syntaxiques peuvent s'acquérir sur le modèle de ce qu'on pratique déjà pour les variations morphologiques. La proposition indicative (travailler en langue oralement sur les constructions verbales) ne vaut pas que pour les classes de maternelle, il est même probable que son importance vaille encore davantage pour les classes de l'élémentaire.

\section{Retour sur l'articulation langue/langage : indicateurs de maitrise et d'apprentissage en cours}

71 Une difficulté majeure réside dans l'identification des indices de corrélation entre une stratégie globale d'acquisition, d'apprentissage langagier et une forme linguistique spécifique. Comment peut-on valider des relations aussi complexes et somme toute invisibles entre des formes langagières actualisées et les procédures (psychologiques) d'actualisation, de construction et d'appropriation?

Une deuxième difficulté tient au point de vue indistinct ou collectif que nous avons adopté : les tours de parole sont envisagés comme significatifs d'un groupe d'apprenants et non pas comme ceux des individus particuliers. Le groupe est défini par l'âge de ses sujets et la nature de l'activité langagière. C'est la forme pronominale qui en tant que telle permet de formuler des hypothèses sur l'apprentissage en cours. 
critères retenir pour interpréter les réalisations pronominales si l'on veut en faire des indicateurs de maitrise et/ou d'apprentissage ? La variété lexicale (selon quel repère, une séance ou un élève particulier?); La variété et la complétude syntaxique? La complexité syntaxique de la construction dans son environnement?

Notre hypothèse a consisté à associer complémentairement une forme complexe et un contenu d'histoire. Il nous a semblé que la grammaire intuitive des élèves les conduisait à des choix d'abord lexicaux et qu'un "enseignement explicite» devrait s'efforcer de renforcer la composante syntaxique des formes en sensibilisant les élèves à la variation des emplois. À titre exploratoire, nous regroupons ci-dessous deux familles de critères et les indicateurs susceptibles de les satisfaire. Nous commençons par les critères langagiers et poursuivons par le domaine linguistique.

\section{Tâches langagières et prises de risque communicationnel}

- Intervention collaborative et indicateurs d'écoute: moments (échelonnés?) des prises de parole au cours de la séance.

- Longueur de l'intervention et indicateurs de réflexion: hésitation, reprise, complétude et réfection des énoncés.

- Pertinence informationnelle : indicateurs d'innovation thématique.

- Enchainement d'énoncés dans une intervention et usage de connecteurs (et, et puis, et après... ).

\section{Réalisations linguistiques illustrées par les verbes pronominaux}

- Variété et ajustement lexical : s'en aller, s'absenter, s'enfuir.

- Forme fléchie et composée : il s'est aventuré dans le potager.

- Variation du sujet : ça se voit qu'il est fatigué, il-lapin et il-bouton.

- Constructions verbales complexes : elle se demande ce qui s'est passé.

- Traitement de la diathèse et du passif : son bouton s'est pris dans le filet ; se faire prendre par...

- Prédicat attributif : il s'est vu (cru) mort quand il a vu arriver M. Grégory.

Les critères et les indicateurs qui précèdent ne sont exploitables que dans une phase de retour sur la séance par le maitre, à l'appui des transcriptions faites. Ils offrent peut-être l'intérêt de pouvoir suivre un élève en particulier. Les indicateurs linguistiques sont classés dans un ordre présumé de difficulté croissante et donc de rareté supposée. La réalisation conjointe des indicateurs équivaut à un progrès avéré. Enfin, les items sont formulés positivement, sans que nous ayons cherché à nommer des emplois erronés.

\section{BIBLIOGRAPHIE}

BARCELó, G. J. \& BRES, J. (2006). Les temps de l'indicatif en français. Gap/Paris : Ophrys.

BÉGUELIN, M.-J., CORMINBOEUf, G. \& JOHNSEN, L. A. (2014). « Réanalyse et changement linguistique : présentation ». Langages 196, p. 3-11. En ligne : https://www.cairn.info/revue-langages-2014-4page-3.htm. 
BLANCHE-BENVENISTE, C. et al. (1984). Pronom et syntaxe. L'approche pronominale et son application au français. Paris : SELAF.

BLANCHE-BENVENISTE, C. (1990). Le français parlé. Études grammaticales. Paris : CNRS Éd.

BLANCHE-BENVENISTE, C. (2010). Le français. Usages de la langue parlée. Leuven/Paris : Peeters.

BOONS, J.-P. (1987). « La notion sémantique de déplacement dans une classification syntaxique des verbes locatifs ». Langue française 76, p. 5-40. En ligne : https://www.persee.fr/doc/

lfr_0023-8368_1987_num_76_1_4729.

BOONS, J.-P., GUILLET, A. \& LECLÈRE, C. (1976). La structure des phrases simples en français. Constructions intransitives. Genève : Droz.

CATACH, N. (1984). Les listes orthographiques de base du français (LOB). Les mots les plus fréquents et leurs formes fléchies les plus fréquentes. Paris : Nathan.

FRANÇOIS, F., HUDELOT, C. \& SABEAU-JOUANNET, É. (1984). Conduites linguistiques chez le jeune enfant.

Paris : Presses universitaires de France.

MARTINOT, C. (2000). «Étude comparative des processus de reformulation chez des enfants de 5 à 11 ans ». Langages 140, p. 92-123. En ligne : https://www.persee.fr/doc/

lgge_0458-726x_2000_num_34_140_2392.

MELIS, L. (1990). La voie pronominale. La systématique des tours pronominaux en français moderne. Louvain-la-Neuve : Duculot.

MOIGNET, G. (1981). Systématique de la langue française. Paris : Klincksieck.

PÉROZ, P. (2010). Apprentissage du langage oral à l'école maternelle. Pour une pédagogie de l'écoute.

Metz : CRDP de Lorraine.

STEFANINI, J. (1962). La voix pronominale en ancien et en moyen français. Thèse en lettres, Université de Paris.

STEFANINI, J. (1992). Linguistique et langue française. Paris : CNRS Éditions.

VASSANT, A. (1980a). « Lexique, sémantique et grammaire dans la voix verbale en français ». In : Straka, G \& Martin, R. (dirs), Hommage à la mémoire de Gérard Moignet. Mélanges de linguistique et de philologie. Strasbourg: Centre de philologie et de littératures romanes, p. 143-172.

VASSANT, A. (1980b). « Pour une étude de l'aspect verbal ». L'information grammaticale 4, p. 12-19. En ligne : https://www.persee.fr/doc/igram_0222-9838_1980_num_4_1_2500.

\section{ANNEXES}

L'épisode du groseillier

SÉANCE 1 (total : 77 tours de parole) 


\begin{tabular}{|l|l|l|l|}
\hline Tour & & & $\begin{array}{l}\text { eh bien quand monsieur Grégory il est arrivé/ il a dit/ Pierre/ qu'est-ce qu'il } \\
\text { y a dans ce potager/ il a vu Pierre/ et monsieur Grégory il a dit je te } \\
\text { transformerai en pâté/ après Pierre il a couru/ et monsieur Grégory il l'a } \\
\text { coursé et après Pierre il a perdu une chaussure dans les choux/ et l'autre } \\
\text { dans les pommes de terre et il s'est accroché dans un légume et après au moment } \\
\text { où il fallait il enlève son gilet/ monsieur Grégory s'est retourné/ il a entendu } \\
\text { atchoum derrière lui/ il passe entre les jambes de monsieur Grégory et après } \\
\text { Pierre il rentre dans la remise }\end{array}$ \\
\hline
\end{tabular}

Tours 63-69

\begin{tabular}{|c|c|c|}
\hline 63 & M & sans rien oublier/ est-ce qu'on a tout raconté \\
\hline 64 & $\mathrm{El}(\mathrm{s})$ & oui \\
\hline 65 & Oudriss & $\begin{array}{l}\text { non/ eh bien Pierre Pierre Pierre/ il a enlevé sa veste/ elle a dit la maman/ t'as } \\
\text { enlevé ta veste/ tu l'as mis où et il répond pas/ il répond pas }\end{array}$ \\
\hline 66 & Shona & $\begin{array}{l}\text { il dit je l'ai perdue dans le pré/ mais quand il faisait chaud alors je l'ai enlevée/ et } \\
\text { il l'a enlevé la veste }\end{array}$ \\
\hline 67 & M & c'est dans l'histoire? \\
\hline 68 & Mourad & non \\
\hline 69 & Daoud & $\begin{array}{l}\text { la chemise de Pierre/ elle était accrochée aux pommes de terre et après il a accroché sur les } \\
\text { pommes de terre et et après/il a enlevé sa chemise et après/ et après il recouru }\end{array}$ \\
\hline
\end{tabular}

SÉANCE 2 (total : 328 tours de parole)

Tours 68-77

\begin{tabular}{|l|l|l|}
\hline 68 & Sandra & $\begin{array}{l}\text { il s'enfuit partout/ il court ventre à terre/ il perd ses chaussures dans les pommes } \\
\text { de terre et l'autre dans les choux// }\end{array}$ \\
\hline 69 & Sima & il XXX \\
\hline 70 & Sandra & il coince son gilet \\
\hline 71 & M & attends elle va le dire/ vas-y Sima elle t'a un peu aidée \\
\hline 72 & Sima & \\
\hline 73 & M & redis-lui Sandra \\
\hline 74 & Sandra & il essaie d'enlever son gilet \\
\hline 75 & M & vas-y Sima \\
\hline
\end{tabular}




\begin{tabular}{|l|l|l|}
\hline 76 & Sima & et il arrivait pas à enlever son gilet \\
\hline 77 & Alice & $\begin{array}{l}\text { il est obligé d'enlever son gilet et de s'enfuir à toute vitesse et puis il se cache dans un } \\
\text { arrosoir }\end{array}$ \\
\hline
\end{tabular}

SÉANCE 3 (total : 433 tours de parole)

Tours 186-213

\begin{tabular}{|c|c|c|}
\hline 186 & M & $\begin{array}{l}\text { ce n'est pas encore tout de suite// mais avant d'être allé dans la remise/ que lui } \\
\text { est-il arrivé }\end{array}$ \\
\hline 187 & Malo & il était dans le jardin de monsieur Grégory \\
\hline 188 & Clarissa & il était accroché sur le fil \\
\hline 189 & M & Clarissa \\
\hline 190 & Clarissa & il était accroché sur le fil/// le bouton::: le bouton \\
\hline 191 & Marianne & il était accroché à le fil \\
\hline 192 & Clarissa & le bouton::: le bouton de son gilet \\
\hline 193 & Marianne & il s'est accroché au filet \\
\hline 194 & Clarissa & le bouton::: son gilet/il est accroché à \\
\hline 195 & Marianne & à un filet \\
\hline 196 & Clarissa & et il a remarqué dans le ciel au-dessus de lui des oiseaux qui le regardaient \\
\hline 197 & Malo & il le cherche partout \\
\hline 198 & Clarissa & il lui dit::: il lui dit/ les oiseaux lui disent/ sauve-toi \\
\hline 199 & M & oui// Yannick/ pourquoi les oiseaux lui disent/ sauve-toi \\
\hline 200 & Yannick & parce que \\
\hline 201 & Melwan & parce que monsieur Grégory/ il va l'attraper \\
\hline 202 & Clarissa & il croit qu'il va l'attraper \\
\hline 203 & M & alors que fait Pierre quand il s'est coincé dans le filet \\
\hline 204 & Viviane & il::: il::: sa maman \\
\hline 205 & M & Viviane \\
\hline 206 & Viviane & il essaie d'enlever dans le filet \\
\hline
\end{tabular}




\begin{tabular}{|l|l|l|}
\hline 207 & Melwan & $\begin{array}{l}\text { la maman/ elle dit/ pourquoi il a perdu tous ses habits encore une fois// ses } \\
\text { chaussures dans les légumes de monsieur Grégory }\end{array}$ \\
\hline 208 & M & et son gilet? \\
\hline 209 & Sam & le gilet/ il a plus son gilet \\
\hline 210 & Melwan & son gilet/ il était accroché au fil \\
\hline 211 & Viviane & au filet \\
\hline 212 & Melwan & au filet \\
\hline 213 & Clarissa & il arrive pas à trouver le trou par où il est entré \\
\hline
\end{tabular}

SÉANCE 4 (total : 242 tours de parole)

Tours 42-57

\begin{tabular}{|l|l|l|}
\hline 42 & M & Judicaël \\
\hline 43 & Judicaël & $\begin{array}{l}\text { il a eu très peur// et après il a couru et après il a perdu une chaussure dans les } \\
\text { pommes de terre et dans les choux et il tourne dans tous les sens et }\end{array}$ \\
\hline 44 & M & Keliane? je ne t'entends pas ma grande \\
\hline 45 & Keliane & et son gilet il est accra accroché au/ à la branche \\
\hline 46 & M & vous êtes d'accord avec Keliane? \\
\hline 47 & Carina & non \\
\hline 48 & M & Carina \\
\hline 49 & Carina & au filet \\
\hline 50 & Thiébaud & au filet des groseilles \\
\hline 51 & Carina & au filet des groseilles// \\
\hline 52 & M & Keliane redis-le \\
\hline 53 & Keliane & son gilet s'attache/ dans les groseilles \\
\hline 54 & Thiébaud & au fil des groseilles \\
\hline 55 & Keliane & au fil des groseilles et il/ il enlève vite son gilet et après il reprend sa course folle \\
\hline 56 & M & oui \\
\hline 50.00 l & et après il y a des oiseaux qui lui disent sauve-toi vite sauve-toi vite \\
\hline
\end{tabular}


SÉANCE 5 (total : 102 tours de parole)

Tour 5

5 Alexis il était coincé dans le filet de groseilles//

Tours 16-23

\begin{tabular}{|l|l|l|}
\hline 16 & Alexis & il a sauté dans l'arrosoir \\
\hline 17 & Emilie & $\begin{array}{l}\text { et il dit euh: monsieur Grégory quand il le voit sale voleur je vais t'attraper/ je } \\
\text { vais te transformer en pâté }\end{array}$ \\
\hline 18 & Alexis & $\begin{array}{l}\text { euh// il était/// il a tout mangé// tout ce qu'il a vu/ il a entendu un bruit } \\
\text { bizarre// }\end{array}$ \\
\hline 19 & Frédéric & il y a un bouton qui est accroché/le bouton de son gilet à Pierre// \\
\hline 20 & Emilie & $\begin{array}{l}\text { et euh les oiseaux quand ils voient Pierre ils lui disent de s'enfuir vite et euh: } \\
\text { Pierre eh ben juste à temps il trouve une solution// }\end{array}$ \\
\hline 21 & M & tu la connais toi la solution? \\
\hline 22 & Emilie & il enlève son manteau \\
\hline 23 & Agatha & Pierre eh ben il enlève son zilet// \\
\hline
\end{tabular}

Tour 70

les chaussures/ il a perdu une chaussure dans les carottes et une chaussure dans les 70 Lili choux/// et son gilet/ il a perdu son gilet/ il était coincé alors il a enlevé son gilet et il a rattrapé sa course//

SÉANCE 6 (total : 113 tours de parole)

Tours 5-17

\begin{tabular}{|l|l|l|}
\hline 5 & Emilie & votre père y est allé une fois et il n'est jamais jamais revenu \\
\hline 6 & Alexis & y avait une ou deux choses qui étaient pas accrochées \\
\hline 7 & M & explique// \\
\hline 8 & Alexis & il a sauté dans l'arrosoir et il s'est pris les les/ fils de groseilles \\
\hline 9 & M & alors toi tu es déjà dans le potager// nous on était avec la maman et ses petits// \\
\hline
\end{tabular}




\begin{tabular}{|l|l|l|}
\hline 10 & Marco & $\begin{array}{l}\text { euh: euh:/ Neige/ Capucine et euh: Romarin ils xxx en train de cueillir des fleurs } \\
\text { mais euh: Pierre il a été dans le jardin de monsieur Grégory }\end{array}$ \\
\hline 11 & Frédéric & c'est pas des fleurs c'est des mûres \\
\hline 12 & Marco & $\begin{array}{l}\text { oui// et après il va dans le jardin et après euh tout il est en train de xxx grignoter } \\
\text { court }\end{array}$ \\
\hline 13 & Emilie & $\begin{array}{l}\text { et euh monsieur Grégory qui approche et après euh et après Pierre qui dit il } \\
\text { pâté }\end{array}$ \\
\hline 14 & Leslie & $\begin{array}{l}\text { et après il perd une chaussure dans les dans les choux et une chaussure dans les } \\
\text { pommes de terre }\end{array}$ \\
\hline 15 & Daly & il s'a accroché son bouton de gilet dans les plants de groseilles \\
\hline 16 & Frédéric & et après les oiseaux ils disent vite vite sauve-toi il y a monsieur Grégory qui vient \\
\hline 17 & Emilie & $\begin{array}{l}\text { après il a trouvé une solution/ il enlève sa veste et il va dans la remise pour aller } \\
\text { dans l'arrosoir et il a fait une erreur parce que l'eau elle était glacée// }\end{array}$ \\
\hline
\end{tabular}

SÉANCE 7 (total : 113 tours de parole)

$\varnothing$

SÉANCE 8 (total : 150 tours de parole)

Tours 43-78

\begin{tabular}{|l|l|l|}
\hline 43 & M & que se passe-t-il dans sa course folle/ \\
\hline 44 & Viviane & ben il prend/ il court en toute vitesse/ \\
\hline 45 & Nathanaël & \\
\hline 46 & Sihame & il court ventre à terre/ \\
\hline 47 & Nathanaël & il court en plus vite possible/ \\
\hline 48 & Ted & euh/ euh près des euh:: \\
\hline 49 & Viviane & près des euh::: // son bouton y s'est attaché/ \\
\hline 50 & Ted & près des euh des sssss \\
\hline 51 & M & Viviane \\
\hline 52 & Viviane & son bouton i(l) s'est attaché à un fil où se trouve euh comment ça s'appelle \\
\hline 53 & Ted & les cerises/ les/ comme c'est les/// comme les petites boules là/ les \\
\hline
\end{tabular}




\begin{tabular}{|c|c|c|}
\hline 54 & Sergueï & et aussi hier il était épuisé \\
\hline 55 & Viviane & il s'est effondu::: dans dans son terrier/ il est tombé et il s'est endormi \\
\hline 56 & M & donc pendant sa course folle \\
\hline 57 & Ted & les cerises \\
\hline 58 & M & les groseilles tu veux dire \\
\hline 59 & Ted & euh ouais c'est des/// groseilles \\
\hline 60 & M & donc pendant sa course folle qu'est-ce qu'il s'est passé// \\
\hline 61 & Viviane & $\begin{array}{l}\text { il a couru à tout tou tou tou tou tou toute vitesse/ ventre à t/// et son bouton il } \\
\text { s'est accroché au au filet quand il courait ventre à terre/ au::: }\end{array}$ \\
\hline 62 & M & comment a il perdu son::: \\
\hline 63 & $\operatorname{El}(\mathrm{s})$ & gilet/ pull \\
\hline 64 & Viviane & ben euh \\
\hline 65 & Tania & il a enlevé/ il l'a enlevé \\
\hline 66 & Sergueï & et aussi son bouton comme il s'est pris au filet \\
\hline 67 & Viviane & c'est quand \\
\hline 68 & Sergueï & $\begin{array}{l}\text { eh ben il a enlevé son gilet parce qu'il était cassé maintenant et il l'a enlevé pour } \\
\text { reprendre la/// pour courir plus vite }\end{array}$ \\
\hline 69 & Viviane & pour reprendre sa course folle/ et Assia elle a même pas parlé! \\
\hline 70 & M & quelqu'un veut ajouter quelque chose sur la perte du gilet? \\
\hline 71 & Viviane & $\begin{array}{l}\text { ben/ ben son bouton il s'est attaché au filet il fallait/ qu'il l'/// les oiseaux/ ils se } \\
\text { regardaient autour de lui/ les oiseaux ils l'ont observé/ ils ont dit/ sauve-toi/ } \\
\text { sauve-toi parce que/// et monsieur Grégory il est arrivé avec un panier dans la } \\
\text { main/ prêt/ il était prêt à faire du pâté et même euh:: }\end{array}$ \\
\hline 72 & Sergueï & et \\
\hline 73 & Viviane & et et ça veut dire qu'il veut le cuisiner? même euh:: \\
\hline 74 & Sergueï & et aussi il s'est sauvé jusqu'à temps quand monsieur Grégory \\
\hline 75 & Viviane & et même/ je sais maintenant \\
\hline 76 & Sergueï & quand il voulait l'attraper ben il s'est sauvé jusqu'à temps \\
\hline 77 & Sihame & $\begin{array}{l}\text { euh euh euh son bouton du gilet il a il était accroché/ et quand il était accroché il } \\
\text { arrivait plus à se sauver et juste à temps il a enlevé son gilet// }\end{array}$ \\
\hline
\end{tabular}




\begin{tabular}{|l|l|l|}
\hline 78 & $\begin{array}{l}\text { euh quand/ comme il avait enlevé son gilet et et l'a vu une mare avec une chatte } \\
\text { prêt à attraper un poisson rouge elle bougeait sa queue de patience et et après } \\
\text { Pierre est tombé dans la douceur de son terrier et s'effondre par terre endormi } \\
\text { et sa maman dit/ euhm comment as-tu enlevé ton gilet ça fait deux jours }\end{array}$ \\
\hline
\end{tabular}

SÉANCE 9 (total : 113 tours de parole)

Tours $10-11$

\begin{tabular}{|l|l|l|}
\hline 10 & Luc & $\begin{array}{l}\text { et Pierre il a pas écouté sa maman et il a mangé un bouquet de persil et des gros } \\
\text { haricots verts et::: }\end{array}$ \\
\hline 11 & Viviane & $\begin{array}{l}\text { monsieur Grégory l'avait un filet et Pierre son bouton était fait attraper au filet et puis les } \\
\text { oiseaux ils ont dit/ Pierre Pierre vite sauve-toi et juste à temps }\end{array}$ \\
\hline
\end{tabular}

Tours 27-29

\begin{tabular}{|l|l|l|}
\hline 27 & Amélie & $\begin{array}{l}\text { le le Pierre il a sauté dans l'arrosoir il a dit/ quelle erreur y avait y avait beaucoup } \\
\text { d'eau dans l'arrosoir et après il a Pierre il a fait atchoum et monsieur Grégory il } \\
\text { s'est retourné }\end{array}$ \\
\hline 28 & Luc & $\begin{array}{l}\text { // Pierre les il s'est attrapé par le bouton parce qu'il était dans le filet et les oiseaux vite } \\
\text { Pierre et Pierre il a sauté dans l'arrosoir et l'avait froid et il a fait atchoum et puis } \\
\text { monsieur Grégory il s'est retourné }\end{array}$ \\
\hline 29 & Vicky & et les oiseaux il a dit/ cours cours cours vite Pierre et Pierre il s'est endormi \\
\hline
\end{tabular}

Tours 108-113

\begin{tabular}{|l|l|l|}
\hline 108 & Amélie & $\begin{array}{l}\text { la maman et elle a donné une tisane de camomille à Pierre et il s'est endormi } \\
\text { Pierre }\end{array}$ \\
\hline 109 & Kader & $\begin{array}{l}\text { Pierre eh ben il avait il est venu la maman elle avait dit/ c'est la dernière fois que } \\
\text { tu perds tes habits tes saussures(chaussures) Pierre il a mis ses saussures } \\
\text { (chaussures) dans les choux et l'autre dans les pommes de terre après le boue il a } \\
\text { enlevé san(son) gilet }\end{array}$ \\
\hline 110 & Maëlyg & $\begin{array}{l}\text { Pierre la maman de Pierre elle a dit/ c'est la deuxième fois que tu as perdu tes } \\
\text { vêtements }\end{array}$ \\
\hline 111 & Vicky & et la maman elle prend un pain et du lait \\
\hline 112 & Julien & et aussi son bouton/le bouton de Pierre de son gilet vient de s'attraper par le fil \\
\hline 113 & Maëlyg & Pierre la maman de Pierre elle a ramené du pain et du lait \\
\hline
\end{tabular}

SÉANCE 10 (185 tours de parole) 
Tours 17-26

\begin{tabular}{|l|l|l|}
\hline 17 & M & Lucie \\
\hline 18 & Lucie & madame Grégory elle a fait de lui un civet \\
\hline 19 & M & Hugues \\
\hline 20 & Hugues & $\begin{array}{l}\text { euh euh: en fait i(l) dit euh monsieur Grégory c'est qui embête ce jardin si } \\
\text { tranquille }\end{array}$ \\
\hline 21 & M & Léa \\
\hline 22 & Léa & eh ben aussi $i l$ était accroché et et euh:/ \\
\hline 23 & M & Ewan \\
\hline 24 & Ewan & $\begin{array}{l}\text { la maman elle lui a donné une tisane de camomille quand il: quand il: quand il } \\
\text { était: il avait perdu ses chaussures et son manteau et son gilet }\end{array}$ \\
\hline 25 & M & Hugues \\
\hline 26 & Hugues & sa maman elle l'a mis dans dans le lit \\
\hline
\end{tabular}

Tours $43-50$

\begin{tabular}{|l|l|l|}
\hline 43 & M & Léo \\
\hline 44 & Léo & xxx en fait \\
\hline 45 & M & Hugues \\
\hline 46 & Hugues & $\begin{array}{l}\text { en fait } \mathrm{i}(\mathrm{l}) \mathrm{i}(\mathrm{l}) \mathrm{i}(\mathrm{l}) / \text { quand les oiseaux il a terminé il s'est pris le filet de ses groseilles/ et } \\
\text { puis après et après il a enlevé vite son gilet }\end{array}$ \\
\hline 47 & M & Lucie \\
\hline 48 & Lucie & les oiseaux ils lui ont dit de s'enfuir vite \\
\hline 49 & M & Léa \\
\hline 50 & Léa & il l'a entendu et il s'est tout de suite retourné \\
\hline
\end{tabular}

SÉANCE 11 (total : 142 tours de parole)

Tours 28-30

\begin{tabular}{|l|l|l|}
\hline 28 & Thiébaud & $\begin{array}{l}\text { quand tout à coup euh monsieur Grégory il il a vu euh Pierre eh ben il a enlevé } \\
\text { son gilet et il reprend sa course folle }\end{array}$ \\
\hline
\end{tabular} 


\begin{tabular}{|l|l|l|}
\hline 29 & M & vous avez d'autres choses à raconter sur l'histoire?/ Théophile \\
\hline 30 & Théophile & $\begin{array}{l}\text { et aussi monsieur/ Pierre quand il est passé eh ben que monsieur Grégory } \\
\text { approchait eh ben tout à coup le fil le bouton de son gilet s'est pris dans le plan de } \\
\text { groseilles les/ il regarde autour de lui/ les oiseaux lui disent de se sauver// } \\
\text { tempsieur Grégory approche un panier à la main prêt à l'attraper/ ouf juste à } \\
\text { arrosoir// sans à enlever són gilet/ Il arrive près d'une mare/ il arrive près d'un } \\
\text { erreur! il est à moitié plein d'une eau glacée// euh euh/ Sélèna }\end{array}$ \\
\hline
\end{tabular}

SÉANCE 12 (total : 125 tours de parole)

Tours 18-22

\begin{tabular}{|l|l|l|}
\hline 18 & Giorgio & il a/ il a// il a enlevé sa veste et il s'est détaché du filet \\
\hline 19 & M & ça il faut bien l'expliquer/ explique bien \\
\hline 20 & Giorgio & ça je sais pas \\
\hline 21 & M & Fabricio explique bien ce que disait Giorgio \\
\hline 22 & Fabricio & \begin{tabular}{l} 
il était accroché son bouton/ il était accroché sur le filet/ après il a enlevé son gilet/ il l'a jété dans la remise/ et après il s'est caché dans l'arrosoir \\
\hline
\end{tabular} \\
\hline
\end{tabular}

Tours 36-39

\begin{tabular}{|l|l|l|}
\hline 36 & Anabelle & et/// et// il est passé par un grillage Pierre pour s'en aller \\
\hline 37 & Fabricio & $\begin{array}{l}\text { moi moi je veux dire quand euh/ il est passé entre les jambes de monsieur } \\
\text { Grégory/// }\end{array}$ \\
\hline 38 & Francesco & il allait presque l'attraper \\
\hline 39 & Jean & $\begin{array}{l}\text { eh ben/ euh/ le filet de groseillier il empêchait les oiseaux de mettre sur le grand } \\
\text { groseillier/ Pierre il a passé finalement peut-être en-dessous et il s'est pris dans le bouton }\end{array}$ \\
\hline
\end{tabular}

\section{NOTES}

1. La version définitive de ce travail a bénéficié des remarques attentives des deux relecteurs anonymes, de M. Delaborde et P. Péroz. Je les remercie très sincèrement, ainsi que P. Gil pour son aide.

2. Soit le tour de parole 4 de la séance 9.

3. On ne peut pas garantir absolument la justesse de la transcription étant donné l'ambiguité phonologique du a : s'accrochait ou s'a accroché?

4. Absent du texte source mais précieux pour sa valeur intégrative (ou résomptive). 
5. Auquel il conviendrait d'ajouter s'effondrer, présent dans le texte source. Il ne figure pas dans notre exemple dans la mesure où il ne remplit qu'une fonction «catalyse » ou secondaire (par rapport à la fonction noyau de "réussir à regagner le terrier »), et joue un rôle plus stylistique que narratif, dramatisant le retour à la maison de Pierre le lapin. Les occurrences de s'effondrer dans le corpus sont rares : S4-109; S8-55 et S8-78.

6. À partir de laquelle il faut procéder à une inférence causale : se débarrasser du gilet implique pour Pierre qu'il puisse reprendre sa course (sans entrave).

\section{RÉSUMÉS}

Étant donné la tâche assignée aux élèves de restituer l'histoire entendue de Danger dans le potager, les séances de langage enregistrées dans 12 classes de maternelle et qui constituent le corpus CLEA peuvent s'analyser doublement: du point de vue de la maitrise linguistique d'une forme sélectionnée et/ou du point de vue de la compétence langagière à rendre compte de l'histoire ou d'un de ses épisodes. L'article a choisi cette double entrée, retenant les tours pronominaux comme analyseur linguistique d'une part, et d'autre part l'épisode du groseillier pour sa complexité référentielle et narrative et le fait qu'il nécessite potentiellement l'usage de verbes pronominaux (se prendre, s'accrocher au filet). Après une entrée en matière sur la méthode et le cadre d'analyse retenus, l'article recense et examine les verbes pronominaux du corpus d'après un classement inspiré de celui de C. Blanche-Benveniste et al. (1984). Ensuite, l'épisode du groseillier qui constitue un obstacle narratif à la fuite du lapin (et à son retour sain et sauf à la maison) est décomposé en quatre temps, à partir desquels se rapportent les verbalisations des élèves. Le fil directeur de ces analyses demeure l'emploi des verbes pronominaux. On a ainsi cherché à dresser, sur la base d'un corpus collectif, l'inventaire d'un tour langagier complexe dont l'apprentissage nécessite d'être mené selon une progression qui n'oublie pas le premier temps des activités orales, répétitives, et réflexives sans être " grammaticales » (les activités de langue dites « décrochées »).

Given the task assigned to pupils of restoring the story heard in The Tale of Peter Rabbit, the language sessions recorded in 12 kindergarten classes that constitute the "CLEA corpus" can be analyzed doubly: from the point of view of the linguistic mastery of a selected form and /or from the point of view of the language competence to account for the story or one of its episodes. The article chose this double entry, retaining the pronominal turns as a linguistic analyzer on the one hand, and, on the other hand, the gooseberry episode for its referential and narrative complexity and the fact that it potentially requires the use of pronominal verbs in French (se faire prendre, to hang on to the net). After an introduction on the method and the framework of analysis retained, the article identifies and examines the pronominal verbs of the corpus according to a ranking inspired by that of C.Blanche-Benveniste and al. (1984). Then, the episode of the gooseberry which constitutes a narrative obstacle to the escape of the rabbit (and to its safe return home) is broken down into four stages,from which the pupils' verbalizations will relate to. The guiding thread of those analyzes remains the use of pronominal verbs. The aim was to draw up,on the basis of a collective corpus, the inventory of a complex linguistic turn whose learning requires to be conducted according to a progression which does not forget the first time / stage ? of the oral activities, repetitive and reflexive without being 'grammatical' ( the so-called 'off-hook' activities). 
INDEX

Mots-clés : s'absenter - s'aventurer - se faire prendre etc., répertoire des tours pronominaux, propriétés syntaxiques, rôles sémantiques, enseignement de la langue

Keywords : s'absenter - s'aventurer - se faire prendre etc., pronominal turns, syntactical properties, semantic roles, language teaching

\section{AUTEUR}

\section{CAROLINE MASSERON}

Université de Lorraine, Crem, EA 3476, F-57000, France 